\title{
Clinical benefit from afatinib in an advanced squamous cell lung carcinoma patient harboring HER2 S3 IOY mutation: a case report
}

This article was published in the following Dove Press journal:

OncoTargets and Therapy

\author{
Yan Gao' \\ Aihong Zheng ${ }^{2}$ \\ Xiuming $\mathrm{Zhu}^{2}$ \\ Jia Song ${ }^{3}$ \\ Qian Xue ${ }^{2}$ \\ 'Department of Western Medicine, \\ Wenxin Community Health Service \\ Center, Hangzhou 310016, China; \\ ${ }^{2}$ The Department of Medical \\ Oncology, Zhejiang Provincial \\ People's Hospital, Hangzhou 310014 \\ China; ${ }^{3}$ The Medical Department. \\ 3D Medicines Inc., Shanghai 20III4, \\ China
}

\begin{abstract}
Background: HER 2 mutations are identified in approximately $2 \%$ of non-small-cell lung cancer (NSCLC) cases and are predominantly observed in non-smokers, females, and adenocarcinoma patients. Although afatinib is recommended for treating NSCLC patients with HER2 mutation, the therapy is most efficacious in patients harboring HER2 exon 20 insertions, especially the in-frame insertion YVMA. Research on the treatment of the extracellular domain mutation is relatively rare.
\end{abstract}

Case presentation: We discuss a 76-year-old Chinese man with a heavy-smoking history who was diagnosed with stage IV squamous cell lung carcinoma. First-line treatment with the angiogenesis inhibitor endostar and systemic chemotherapy with docetaxel plus cisplatin were administered, but the patient ceased treatment because of chemotherapy-induced adverse events. Based on the test result from an amplification refractory mutation system PCR, EGFR-inhibitor icotinib was prescribed, but there was still no evidence of a response. Then, next-generation sequencing identified an HER2 S310Y mutation, and afatinib therapy resulted in a gradual, but substantial reduction in tumor size.

Conclusion: This is the first published case report of the successful management of HER2 S310Y mutation squamous cell lung carcinoma with afatinib. Considering the fact that this rare HER 2 mutation clinically benefited from afatinib treatment, attention should be paid to the incidence of HER2 in NSCLC patients with inconsistent histological characteristics compared with those previous published. With the guidance of a precise diagnosis, we should realize the significance of other HER2 gene mutations and next-generation sequencing as a diagnostic method.

Keywords: afatinib, HER2 S310Y, squamous cell lung carcinoma

\section{Introduction}

Lung cancer is the leading cause of cancer-related mortality worldwide, and nonsmall-cell lung cancer (NSCLC) accounts for over $85 \%$ of lung cancers and represents a major histological subtype. ${ }^{1,2}$ Almost half of the patients are diagnosed during stage IV NSCLC, and conventional systemic chemotherapy is the cornerstone of treatment. However, a modest benefit is provided in survival and the overall 5-year survival rate of NSCLC is still low. ${ }^{1}$ For advanced NSCLC, immune checkpoint inhibitors (ICIs) have been approved by the US Food and Drug Administration based on Phase III trials in first- or beyond line treatments. ${ }^{3-6}$ Unfortunately, the major concern regarding immunotherapy in NSCLC is cost-effectiveness. As we all know, immunotherapy is a high-cost treatment, and the efficacy rate of ICI monotherapy is relatively low. ${ }^{7,8}$ Moreover, driver gene mutations might be implicated in the shorter
Correspondence: Qian Xue

The Department of Medical Oncology, Zhejiang Provincial People's Hospital, I58 Shangtang Road, Hangzhou 310014 , China

$\mathrm{Tel}+86 \quad$ I35 88498846

Fax +86 57I 85239988

Email 13588498846@I63.com (c)
hereby accept the Terms. Non-commercial uses of the work are permitted without any further permission from Dove Medical Press Limited, provided the work is properly attributed. For permission hereby accept the Terms. Non-commercial uses of the work are permitted without any further permission from Dove Mediect
for commercial use of this work, please see paragraphs 4.2 and 5 of our Terms (https://www.dovepress.com/terms.php). 
progression-free survival (PFS) and the low response rate. ${ }^{9}$ Based on these reasons, limitations exist in the application of immunotherapy.

With the development of molecular biology, the utilization of tyrosine kinase inhibitors (TKIs) has unveiled a new era for NSCLC treatment. HER2, also known as ErbB2, a member of the ErbB receptor tyrosine kinase family, is a major proliferative driver that activates downstream signaling, such as the PI3K-Akt and MEK-ERK pathways. A clinical characteristics analysis revealed that HER2 mutations were more frequently observed in female patients who never smoked, and had an adenocarcinoma histology. ${ }^{10-12}$ HER2 mutations occur in approximately $2 \%$ to $4 \%$ of NSCLC, and the main mutation types of HER2 include protein overexpression, gene amplification, in-frame insertion in exon 20, and point mutation. ${ }^{13}$ In a prior report, exon 20 insertion mutations, especially a $12 \mathrm{bp}$ insertion mutation causing the duplication of the amino acids YVMA at codon 775, were the most common mutation type (over $80 \%$ ). ${ }^{11}$ Afatinib, an oral HER family blocker, covalently binds and irreversibly blocks all kinase-competent HER family members. ${ }^{14}$ Moreover, afatinib therapy has been reported to display promising results and a manageable toxicity profile by targeting mutations in exon 20, such as p.Ala775-Gly776 ins, p.Tyr772-Ala775dup, p.Gly776Leu, and p.Gly778-Pro780 dup, in several studies on NSCLC. ${ }^{13,15-17}$

Compared with the well-characterized exon 20 mutations, mutations in extracellular domain (ECD) have been poorly investigated. The HER2 S310 single site substitution, located in the HER2 ECD, has been verified to be oncogenic. An in vitro study confirmed that the HER2 S310Y mutant promotes non-covalent dimerization and kinase activation and supports robust colony formation in soft agar. After treatment with HER2 inhibitors afatinib, lapatinib, and neratinib, Ba/F3 cells harboring HER2 S310Y mutant exhibited increasing sensitivity to the inhibitors compared to cells expressing the kinase domain mutant (ins YVMA) or the wild-type HER $2 .{ }^{18}$ Clinically, there has been a report of a patient with lung adenocarcinoma harboring the HER2 S310Y mutation who was treated with afatinib. ${ }^{19}$ After the afatinib treatment, the patient achieved a partial response (PR) in the chest lesions and an almost complete response in the intracranial lesions and had a PFS of 5 months. The case was the first clinical evidence of effective management by afatinib targeting the HER2 S310Y mutation in lung adenocarcinoma. However, among the reported lung squamous cell carcinoma (SqCC) biomarkers, HER2, as a target, remains poorly described. Herein, we published a rare case of a lung SqCC patient with an HER2 S310Y mutation who responded to afatinib.

\section{Case presentation}

A 76-year-old Chinese male, with a history of heavy smoking (30 pack-years), presented to a local hospital due to continuous coughing and phlegm in September 2016. A chest computed tomography (CT) scan revealed a space-occupying lesion in the left lower lobe and left pleural effusion, and central type lung cancer was considered. In May 2017, the patient was referred to our hospital for further examination. CT scan identified several solitary lumps in the left lower lobe, and the patient also had mediastinal lymphadenopathy and pleural metastasis (Figure 1). An immunohistochemistry (IHC) examination of the transbronchial needle aspiration biopsy specimen revealed that the tumor cells exhibited a strong positive staining for CK5/6, P40, and P63 (Figure 2). Based on the IHC examination, the patient was diagnosed with stage IV squamous cell lung carcinoma (pT4N3M1). According to the patient's wishes and the exclusion of treatment contraindications, the patient underwent treatment with endostar (a novel recombinant human endostatin) combined with chemotherapy: docetaxel and cisplatin. Though the clinical effect assessment showed a PR, the patient ceased treatment due to emerging chemotherapy-induced side effects, namely supraventricular tachycardia. Then, amplification refractory mutation system PCR, to assess the tissue biopsy for EGFR exon 18-21, showed a deletion mutation in exon 19 of $E G F R$ (Figure 3), prompting the initiation of the EGFR-inhibitor icotinib (125 mg tid). A month later, a CT scan revealed a slight enlargement of the primary mass and multiple nodules in the lung compared with the day before the icotinib treatment (Figure 1B and C). Based on the unsatisfactory therapeutic effects, the patient received docetaxel and cisplatin again. In order to establish effective therapy and re-evaluate the molecular features, DNA was extracted from the fresh puncture sample and was subjected to DNA sequencing analysis using next-generation sequencing (NGS). The result showed that the patient had HER 2 exon $8 \mathrm{~S} 310$ Y mutation (c.929C $>$ A) with an allelic fraction of $42.49 \%$ and wild-type $E G F R$ (Figure $3 \mathrm{~B}$ ). Thus, afatinib administration began on November 20, 2017 after prior therapy showed no clinical response. Shortly after the afatinib treatment, the patient experienced rapid clinical symptom relief. After 1 month of the afatinib therapy, a repeated CT scan demonstrated a slight decreased tumor size in the left lobe (Figure 1D and E). According to the Response Evaluation Criteria in Solid Tumors guidelines (version 1.1), 

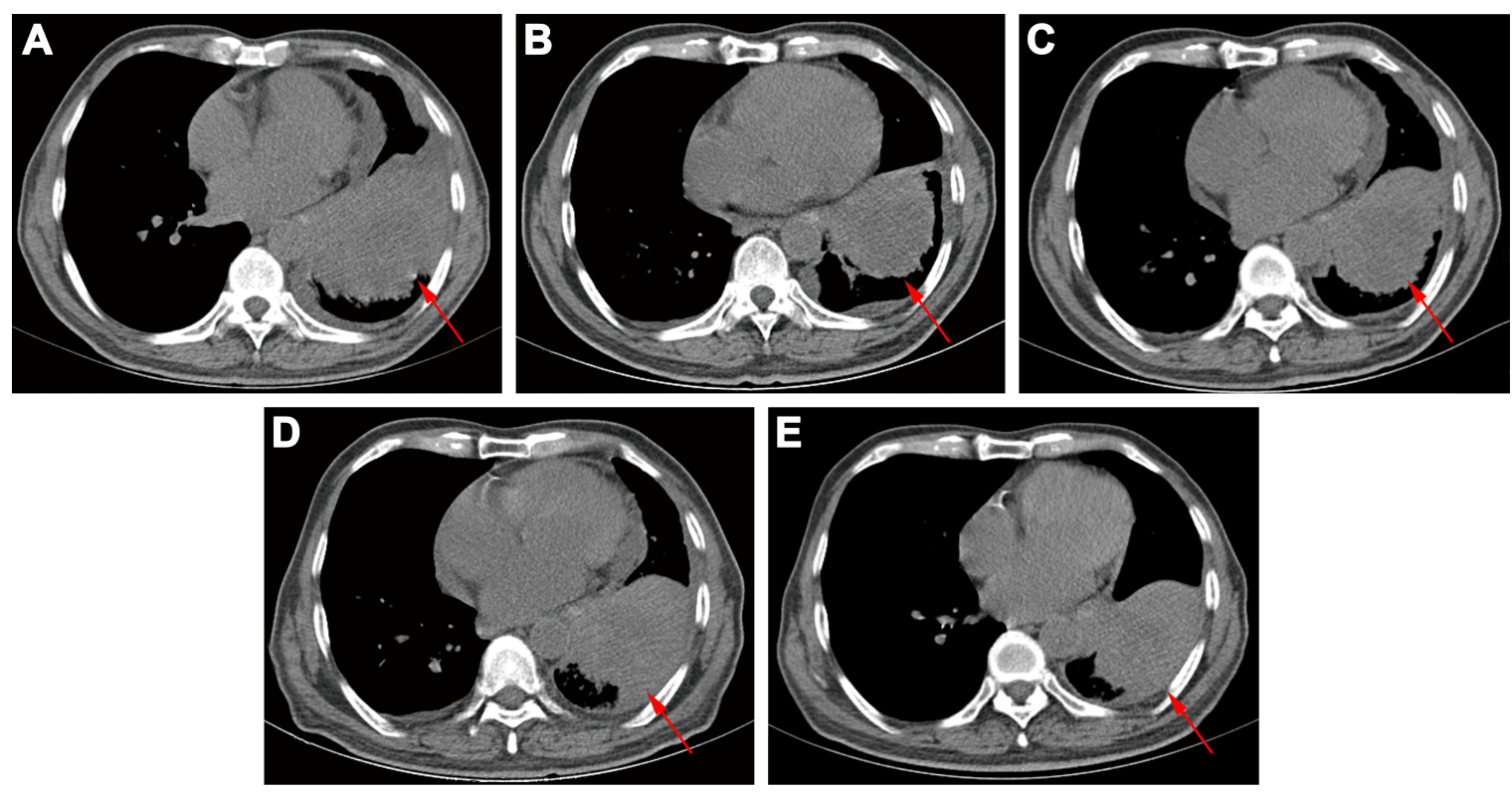

Figure I Computed tomography (CT) scans after different therapies.

Notes: (A) Before any treatment; (B) I day before icotinib treatment; (C) CT of the chest showing a slight increase in tumor volume after I month of icotinib; (D) before afatinib treatment; (E) CT of the chest showing stable disease after I month of afatinib.

the patient was considered to have stable disease (SD) in response to afatinib. During the treatment with afatinib, there were no treatment-related adverse events, including no abnormal hepatic and renal functions. Thus far, after 8 months, no progressive disease has been observed in the patient and he is continuing the treatment with afatinib.

This study was approved by the Medical Ethics Committee of the Zhejiang Provincial People's Hospital. Written informed
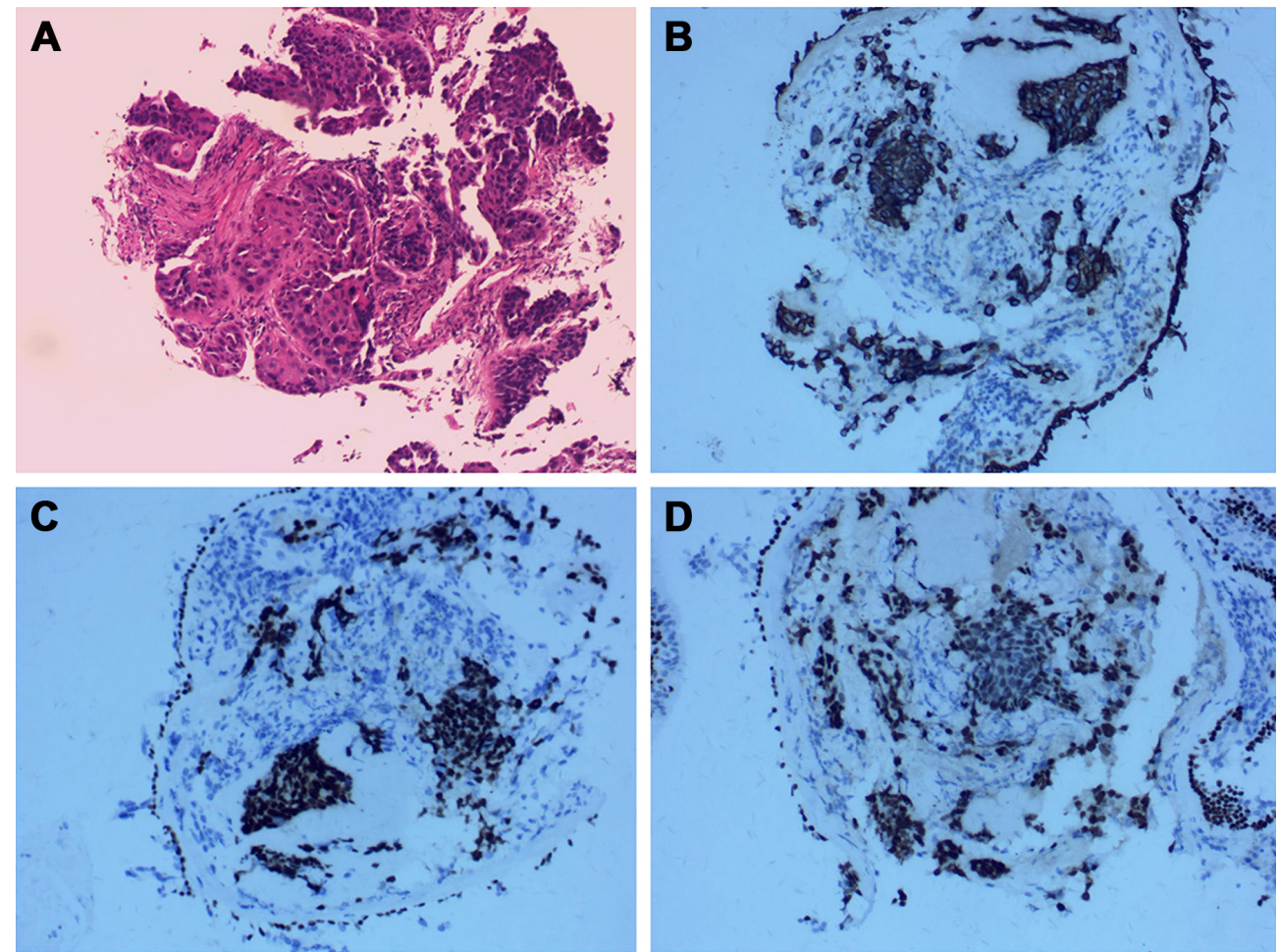

Figure 2 Immunohistochemistry (IHC) of biopsy specimen.

Notes: (A) A high-power magnification of the tumor specimen shows squamous carcinoma (200X). IHC analysis revealed that the lung tumor cells were positive for CK5/6 (B), P40 (C), and P63 (D); 200x. 
A

Amplification plot

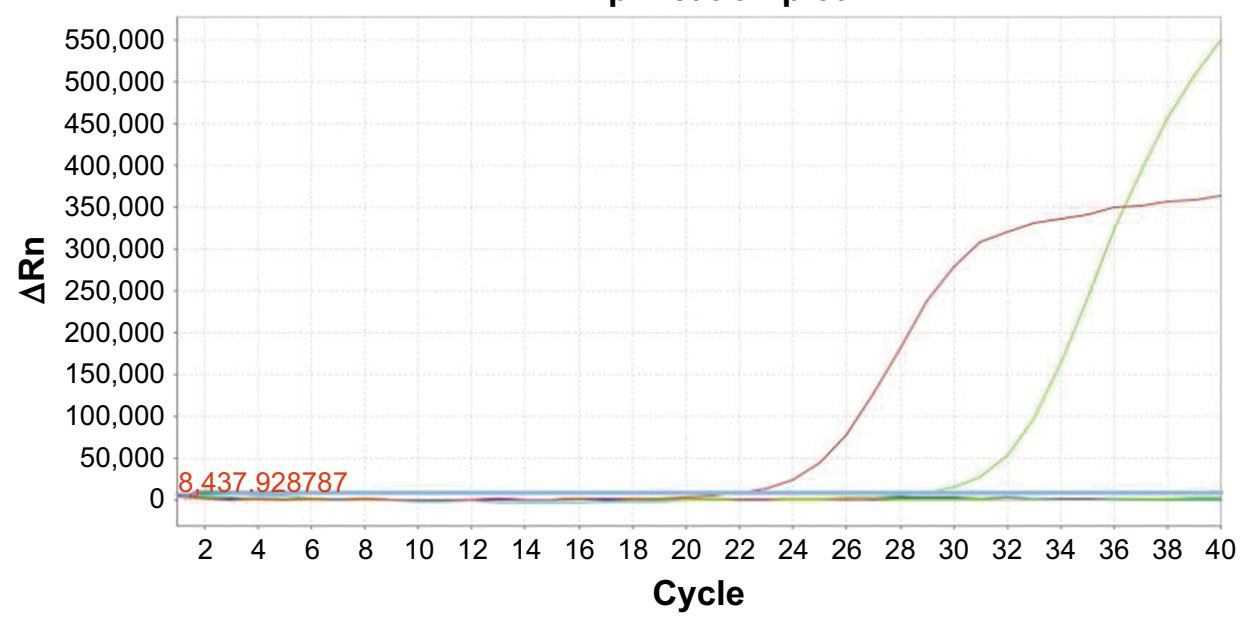

$\square \mathrm{A}-\mathrm{B} \square \mathrm{C} \square \mathrm{D} \square \mathrm{E} \square \mathrm{F} \square \mathrm{G} \square \mathrm{H}$

B

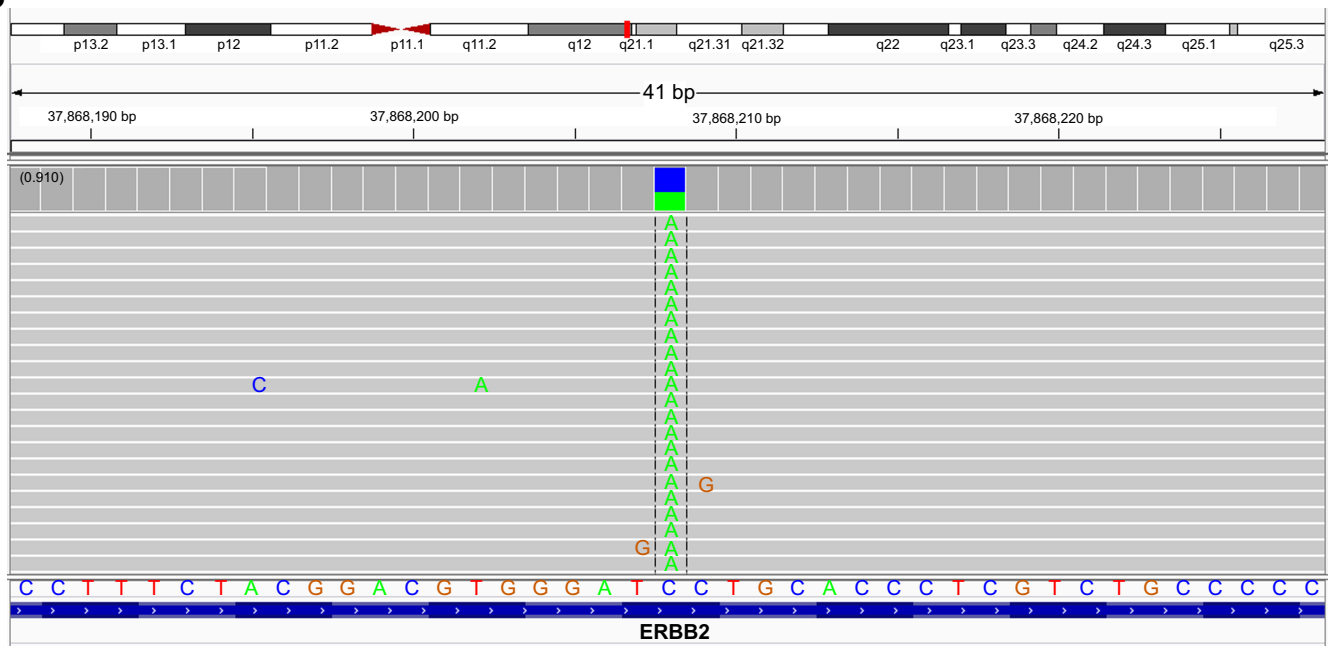

Figure 3 Molecular analysis of gene detection.

Notes: (A) Tissue biopsy showed a deletion mutation in EGFR exon 19 by amplification refractory mutation system PCR. (B) The Integrative Genomics Viewer snapshot of HER2 S3IOY by next-generation sequencing.

consent has also been provided by the patient for publishing the case details and accompanying images in the case study.

\section{Discussion}

At present, there are various treatment regimens for NSCLC, including chemotherapy, immunotherapy, and targeted therapy. Based on this patient's treatment history, he did not have any ICI. Besides the high price, a driver gene mutation was detected by NGS. Despite the observed improved overall survival of NSCLC patients treated with ICIs, the efficacy varies greatly among different immune and molecular profiles of tumors. Particularly, the clinical significance of ICIs for oncogene-driven NSCLC is controversial. ${ }^{20}$ The ImmunoTarget trial indicated that the response of patients carrying the HER2 mutation was rather low and merely reached a $7 \%$ overall response rate. ${ }^{21}$ Based on these reasons, ICIs were not considered.

With the increasing development of targeted therapies, TKIs have been dramatically revolutionized and are widely utilized for lung cancer patients with driver gene mutations. HER2 inhibitors, such as trastuzumab emtansine (T-DM1) and afatinib, are recommended for treatment of NSCLC by the National Comprehensive Cancer Network (NCCN) guidelines. However, the benefiting crowd of T-DM1 is HER2 positive patients whose detecting result was $3+$ by $\mathrm{IHC}^{22}$ and afatinib is preferred for patients with exon 20 insertion mutations. ${ }^{13}$ The variant forms mentioned previously mainly refer to HER2 protein overexpression, copy number 
amplification, and HER2 exon 20 insertion mutations. To date, a limited number of targeted therapy studies focusing on HER2 ECD mutations have been published.

HER $2 \mathrm{~S} 310 \mathrm{Y}$ is a single base mutation in the ECD region, and basic research has proven that this variant activates the downstream signaling pathways. ${ }^{18}$ In breast cancer, two non-HER2-amplified cases harboring the HER2 $\mathrm{S} 310 \mathrm{~F}$ mutation have been reported. The two patients were respectively treated with trastuzumab in combination with endocrine therapy or pertuzumab and endocrine therapy, and both achieved a prolonged response. ${ }^{23,24}$ A metastatic lung adenocarcinoma patient harboring EGFR L858R and ERBB2 S310F mutations was treated with afatinib and achieved a rapid, complete, and durable response both clinically and radiographically. ${ }^{25}$ Moreover, a lung adenocarcinoma case harboring the HER2 S310Y mutation who was treated with afatinib has been reported. ${ }^{19}$ In that case, the patient experienced a longer response duration with afatinib treatment, providing clinical evidence for its use in lung adenocarcinoma. However, to the best of our knowledge, this is the first case report to publish the successful management of HER $2 \mathrm{~S} 310 \mathrm{Y}$ mutation lung SqCC with afatinib. As previously reported, NSCLC patients harboring the HER 2 mutation are mainly female non-smokers with the adenocarcinoma subtype. ${ }^{26}$ This case suggests that HER2 testing should not be merely guided by clinical characteristics. And for patients who do not harbor the genes mentioned by the NCCN guidelines, NGS might be a good option for compiling a treatment plan.

\section{Conclusion}

We, herein, reported the case of a lung SqCC patient harboring the rare HER2 S310Y mutation, who responded to afatinib monotherapy. Based on this finding, afatinib might be considered as an optimal treatment option for lung SqCC patients harboring this type of HER2 mutation profile. Further analysis of the efficacy of afatinib in such cases will lay the foundation for developing optimized treatment protocols for lung SqCC patients with uncommon driver gene mutations.

\section{Acknowledgment}

This work was supported by a grant from the State Administration of Traditional Chinese Medicine of Zhejiang Provincial: NO.0047751.

\section{Author contributions}

YG and JS drafted the manuscript. AHZ and XMZ participated in the collation of the clinical data. QX critically revised the paper. All authors contributed to data analysis, drafting and revising the article, gave final approval of the version to be published, and agree to be accountable for all aspects of the work.

\section{Disclosure}

The authors report no conflicts of interest in this work.

\section{References}

1. American Cancer Society. Cancer Facts \& Figures, 2013. Atlanta, GA: American Cancer Society; 2013.

2. Heist RS, Engelman JA. SnapShot: non-small cell lung cancer. Cancer Cell. 2012;21(3):448.e2.

3. Borghaei H, Paz-Ares L, Horn L, et al. Nivolumab versus Docetaxel in advanced nonsquamous non-small-cell lung cancer. $N$ Engl $J$ Med. 2015;373(17):1627-1639

4. Brahmer J, Reckamp KL, Baas P, et al. Nivolumab versus Docetaxel in advanced squamous-cell non-small-cell lung cancer. $N$ Engl J Med. 2015;373(2):123-135.

5. Herbst RS, Baas P, Kim DW, et al. Pembrolizumab versus docetaxel for previously treated, PD-L1-positive, advanced non-small-cell lung cancer (KEYNOTE-010): a randomised controlled trial. Lancet. 2016; 387(10027):1540-1550.

6. Garon EB, Rizvi NA, Hui R, et al. Pembrolizumab for the treatment of non-small-cell lung cancer. N Engl J Med. 2015;372(21):2018-2028.

7. Aguiar P, de Mello R, Tadokoro H, et al. O.03: Cost Effectiveness of Immune Checkpoint Inhibitors in Non-Small Cell Lung Cancer Relative to PD-L1 Expression. J Thorac Oncol. 2016;11(10S):S169-S170.

8. Jiang T, Zhou C. The past, present and future of immunotherapy against tumor. Transl Lung Cancer Res. 2015;4(3):253-264.

9. Gainor JF, Shaw AT, Sequist LV, et al. EGFR mutations and ALK rearrangements are associated with low response rates to PD-1 pathway blockade in non-small cell lung cancer: A retrospective analysis. Clin Cancer Res. 2016;22(18):4585-4593.

10. Shigematsu H, Takahashi T, Nomura M, et al. Somatic mutations of the HER2 kinase domain in lung adenocarcinomas. Cancer Res. 2005; 65(5):1642-1646.

11. Arcila ME, Chaft JE, Nafa K, et al. Prevalence, clinicopathologic associations, and molecular spectrum of ERBB2 (HER2) tyrosine kinase mutations in lung adenocarcinomas. Clin Cancer Res. 2012;18(18): 4910-4918.

12. Tomizawa K, Suda K, Onozato R, et al. Prognostic and predictive implications of HER2/ERBB2/neu gene mutations in lung cancers. Lung Cancer. 2011;74(1):139-144.

13. Mazières J, Peters S, Lepage B, et al. Lung cancer that harbors an HER2 mutation: epidemiologic characteristics and therapeutic perspectives. J Clin Oncol. 2013;31(16):1997-2003.

14. Solca F, Dahl G, Zoephel A, et al. Target binding properties and cellular activity of afatinib (BIBW 2992), an irreversible ErbB family blocker. $J$ Pharmacol Exp Ther. 2012;343(2):342-350.

15. de Grève J, Teugels E, Geers C, et al. Clinical activity of afatinib (BIBW 2992) in patients with lung adenocarcinoma with mutations in the kinase domain of HER2/neu. Lung Cancer. 2012;76(1):123-127.

16. Li BT, Lee A, O'Toole S, et al. HER2 insertion YVMA mutant lung cancer: Long natural history and response to afatinib. Lung Cancer. 2015;90(3):617-619.

17. Gow CH, Liao WY, Liu YN, Shih JY. Discordant HER2 exon 20 mutation status determines a differential sensitivity to afatinib. $J$ Thorac Oncol. 2015;10(7):e58-e60.

18. Greulich H, Kaplan B, Mertins P, et al. Functional analysis of receptor tyrosine kinase mutations in lung cancer identifies oncogenic extracellular domain mutations of ERBB2. Proc Natl Acad Sci U S A. 2012; 109(36): 14476-14481. 
19. Wang J, Wen Y, Ding G, et al. Efficacy generated by afatinib in a lung adenocarcinoma patient harboring HER2 S310Y mutation. Cancer Biol Ther. 2018;19(6):450-453.

20. Miura Y, Sunaga N. Role of immunotherapy for oncogene-driven nonsmall cell lung cancer. Cancers. 2018;10(8):E245.

21. Julien M, Alexander D, Laurent M. Efficacy of immune-checkpoint inhibitors (ICI) in non-small cell lung cancer (NSCLC) patients harboring activating molecular alterations (ImmuneTarget). J Clin Oncol. 2018;36(5_suppl):172.

22. Stinchcombe T, Stahel RA, Bubendorf L, et al. Efficacy, safety, and biomarker results of trastuzumab emtansine (T-DM1) in patients (pts) with previously treated HER2-overexpressing locally advanced or metastatic non-small cell lung cancer (mNSCLC). J Clin Oncol. 2017;35(15_Suppl):8509.

23. Jasra S, Opyrchal M, Norton L, Mehta R. A rare case of S310F somatic ERBB2 mutation in a HER2-nonamplified breast cancer. Clin Breast Cancer. 2017;17(1):e37-e41.
24. Chumsri S, Weidler J, Ali S, et al. Prolonged response to trastuzumab in a patient with HER2-nonamplified breast cancer with elevated HER2 dimerization harboring an ERBB2 S310F mutation. J Natl Compr Canc Netw. 2015;13(9):1066-1070.

25. Jia Y, Ali SM, Saad S, Chan CA, Miller VA, Halmos B. Successful treatment of a patient with Li-Fraumeni syndrome and metastatic lung adenocarcinoma harboring synchronous EGFR L858R and ERBB2 extracellular domain S310F mutations with the pan-HER inhibitor afatinib. Cancer Biol Ther. 2014;15(8):970-974.

26. Li C, Sun Y, Fang R, et al. Lung adenocarcinomas with HER2-activating mutations are associated with distinct clinical features and HER2/EGFR copy number gains. $J$ Thorac Oncol. 2012;7(1):85-89.
OncoTargets and Therapy

\section{Publish your work in this journal}

OncoTargets and Therapy is an international, peer-reviewed, open access journal focusing on the pathological basis of all cancers, potential targets for therapy and treatment protocols employed to improve the management of cancer patients. The journal also focuses on the impact of management programs and new therapeutic agents and protocols on

\section{Dovepress}

patient perspectives such as quality of life, adherence and satisfaction The manuscript management system is completely online and includes a very quick and fair peer-review system, which is all easy to use. Visit http://www.dovepress.com/testimonials.php to read real quotes from published authors. 